\title{
A genetic analysis of the relationship between life-history variation and heat-shock tolerance in Drosophila buzzatii
}

\author{
ROBERT A. KREBS $\dagger^{*} \&$ VOLKER LOESCHCKE + \\ $\dagger$ Department of Biological, Geological and Environmental Sciences, Cleveland State University, 2399 Euclid Ave., \\ Cleveland, Ohio 44115, U.S.A. and $\$$ Department of Ecology and Genetics, University of Aarhus, \\ Ny Munkegade, Bldg. 540, DK - 8000 Aarhus C, Denmark
}

\begin{abstract}
Although exposure to environmental stress is common in most populations, and the physiological effects of stress on individuals are well studied, the evolutionary importance of stress to populations is not well understood. To address multitrait responses to environmental change and potential constraints on character evolution, we analysed, in 100 isofemale lines of Drosophila buzzatii, the genetic relationships between resistance to a short heat shock and several life-history traits: survival in benign conditions, larval developmental time, fecundity and longevity. Estimates of heritability of larval thermotolerance were low, but significant, and all life-history traits varied significantly among isofemale lines. Several of these traits covaried significantly. Most correlations indicated positive lifehistory relationships, but males and females from lines where female fecundity was higher developed more slowly in the absence of stress, which is a negative life-history relationship. The stress reduced or negated many trait associations, and showed one additional relationship; more larvae from lines that developed fast at $25^{\circ} \mathrm{C}$ survived to adult after stress than did larvae from slow developing lines. These shifts in fitness relationships, when a single stress bout is applied, suggest that even small increases in environmental stress can have profound effects on evolutionary relationships among life-history traits.
\end{abstract}

Keywords: acclimation, developmental time, environmental stress, life table, longevity, trade-offs.

\section{Introduction}

Heterogeneity in the environment can increase genetic variation (Hedrick, 1986), but empirical evidence for this is sometimes contradictory (Hoffmann \& Parsons, 1997; Sgro \& Hoffmann, 1998). We need to identify how specific changes in the environment can quantitatively affect variation in multiple traits. To do this, studies must include defined environmental perturbations. Altering temperature provides one possible means to reach this goal, because many traits vary with the thermal environment (David et al., 1983), performance declines rapidly when temperatures reach some threshold above which stress ensues (Huey \& Bennett, 1990), and temperatures that cause stress are environmentally relevant for many organisms, including Drosophila (Feder, 1996). Furthermore, some molecular changes

*Correspondence. E-mail: r.krebs@popmail.csuohio.edu induced by stress are perhaps better understood than are their functional effects on the phenotype; exposure to short-term heat stress causes a sharp increase in the concentration of specific 'heat-shock' proteins (Hsps), and down-regulation of many others (Parsell \& Lindquist, 1993; Johnston \& Bennett, 1996; Bijlsma \& Loeschcke, 1997), a process often called the heat-shock response. These regulatory changes increase thermotolerance yet minimally alter patterns of growth (Solomon et al., 1991).

Thermotolerance is a plastic trait, however, which is induced by exposure to a nonlethal temperature, and it may impose costs both on rate of development and on fecundity (Krebs \& Loeschcke 1994; Coleman et al., 1995; Hoffmann, 1995). Indeed, the ability to become more thermotolerant and specifically to express higher quantities of one heat-shock protein can reduce larval survival to adulthood in Drosophila melanogaster (Krebs \& Feder, 1997a,b). In contrast, unexpected benefits can be found to be associated with the thermotolerant 
phenotype, as when the induction of thermotolerance and a higher concentration of one heat-shock protein, Hsp70 in D. melanogaster, increase adult longevity (Tatar et al., 1997). Therefore, genetic variation in induction of the stress response may, in part, underlie physiological relationships among life-history traits.

We examined cross-environment correlations among traits in larval and in adult $D$. buzzatii that were either exposed or not exposed to a single bout of heat stress. This species is widely distributed in hot environments where it feeds and breeds in necroses of prickly pear cactus. To obtain sufficient numbers of individuals to test multitrait genetic relationships, 100 recently collected isofemale lines were maintained at low density $(\approx 10$ 15 adults in each of four vials) and were tested for each trait. Multi-vial rearing techniques are recommended by Latter \& Mulley (1995) to delay changes associated with laboratory adaptation, and thereby to preserve the original variation and to minimize the consequences of inbreeding, which are minor during the first five generations when rearing isofemale lines (Hoffmann \& Parsons, 1988). Trait assays therefore began, after just three laboratory generations, with an analysis of heritability for adult thermotolerance (results presented in Krebs \& Loeschcke, 1997), and then proceeded with genetic analyses of larval thermotolerance, larva-toadult survival in the absence of stress and developmental time both after a high temperature treatment and under benign conditions (generation 5). The experiments on trait correlations then concluded with assays of early life fecundity (generation 7) and longevity (generation 8).

\section{Materials and methods}

Collection and rearing methodologies of the Tenerife D. buzzatii lines used here were reported earlier (Krebs \& Loeschcke, 1997) in a study of adult thermotolerance. The 100 isofemale lines, each derived from one male and one virgin female, were split into sets of 25 lines for independent but concurrent analyses that were separated temporally by $3-5$ days to facilitate maintenance and experimental handling. Therefore, any day-to-day variation that might have affected results on flies treated at different times could be identified from differences among these independent sets of lines.

\section{Larval survival and developmental time}

To assay stress resistance of $D$. buzzatii larvae, we harvested eggs on a yeast paste medium from about 100 fourth generation adults from each line. The following day, eggs were rinsed of yeast and placed in a Petri dish with agar until hatching ( $\approx 34 \mathrm{~h}$ after being laid), and 40 1-2 h-old larvae were transferred to each of eight vials per line, where possible. A few lines produced insufficient numbers of larvae. Larvae in four of these replicates per line developed at a constant $25^{\circ} \mathrm{C}$ (no stress), and four others were exposed to a stress of $39^{\circ} \mathrm{C}$ for $6 \mathrm{~h}$ after $24 \mathrm{~h}$ development, and then completed development to the adult stage at a constant $25^{\circ} \mathrm{C}$. Larvae therefore received this heat treatment as late first-instars or early second-instars. The number of adults emerging from each vial was recorded daily to quantify developmental time and survival, and each vial became the unit of replication for statistical analysis.

\section{Early fecundity}

Virgin females were collected for each line. After 3 days at $25^{\circ} \mathrm{C}$, eight females from each line were isolated individually in a food vial with two males obtained from a mass population created previously from these same lines. These flies were subsequently transferred to new food vials after 2 days and were then discarded after 2 additional days. The $6 \mathrm{~cm}^{3}$ Carolina Instant Drosophila medium $(+8 \mathrm{~mL}$ water) that we used is sufficient to rear more offspring than one female could produce over two days. Total emergence numbers therefore provided an estimate of early life fecundity per female, and the average for the eight females gave an estimate for each line.

\section{Adult longevity}

Separately for all lines, 10 replicate vials were collected, each containing 10 males and 10 females at 1-day of age (for a total of 100 males and 100 females per line), and maintained at a constant $25^{\circ} \mathrm{C}$. Mortality was assessed twice weekly, either on Monday and Thursday, or on Tuesday and Friday, determined by the set of vials, at which time flies were transferred to fresh vials of food. We repeated this procedure until all flies died.

\section{Analysis}

The overall design compared variation in seven traits: larval survival and developmental time at a constant $25^{\circ} \mathrm{C}$, these same traits after exposure to a thermal stress treatment, adult survival after heat stress (results from Krebs \& Loeschcke, 1997), fecundity at $25^{\circ} \mathrm{C}$ and longevity at $25^{\circ} \mathrm{C}$. All analyses were repeated in four independent sets of 25 randomly drawn isofemale lines, from which the means were estimated for heritability of larval thermotolerance and for each correlation between traits. Gender effects were separated in all results except fecundity of adult females. Thus correlation analyses compared traits measured for same-sex individuals, except for comparisons involving female fecundity. 
Not all comparisons were strictly independent, however, because within each replicate males and females shared a common larval rearing environment, and males and females were housed together in the longevity analysis.

The genetic basis of stress resistance can be quantified from a mixed-model ANOVA (Hoffmann \& Parsons, 1988; Krebs \& Loeschcke, 1997). For survival and developmental time, the statistical interaction between the fixed treatment effect, stress vs. nonstress, and the random line effect estimate variation in the response of larvae to stress (Barker, 1992). The additive genetic component of variance $\left(S_{\mathrm{A}}^{2}\right)$ is then computed as the interaction mean square minus MSE, all divided by the replicate number $(k=4)$. The intraclass correlation then is $S_{\mathrm{A}}^{2} /\left(S_{\mathrm{A}}^{2}+\mathrm{MSE}\right)$, from which $H$ and $h^{2}$ are determined after accounting for the effect of group size within each vial (Hoffmann \& Parsons, 1988).

\section{Results}

This study observed the effect of heat stress on the relationships among six variables: survival and developmental time of larvae after heat shock, and, at a constant $25^{\circ} \mathrm{C}$, larva-to-adult survival, larval developmental time, adult female fecundity, and adult longevity. These six variables also were compared with adult thermotolerance data, which was measured in these lines two generations earlier than was larval thermotolerance (Krebs \& Loeschcke, 1997).

The heat shock reduced larva-to-adult survival at $25^{\circ} \mathrm{C}$ from $65.7 \pm 6.8 \%$, to $34.0 \pm 7.6 \%$. Stress also lengthened developmental time by about $6 \mathrm{~h}(0.28$ of a day), from a mean of $14.35 \pm 0.19$ days at a constant $25^{\circ} \mathrm{C}$ to $14.62 \pm 0.11$ days for the larvae emerging after the heat shock. This time difference did not significantly exceed the duration of the stress exposure.

\section{Genetic analysis of thermotolerance}

Tolerance of larvae to heat shock varied genetically (Table 1). Significantly positive treatment by line effects, from which additive components of variance are derived, were found in sets one, two and three, but not in set four, although this interaction was significant for developmental time in this fourth set. Combining all lines together, where each group of 25 lines was nested within a variable set, similarly indicated significance of the treatment $\times$ line interaction $\left(F_{93,511}=6.55, P<0.001\right.$, for survival and $F_{93,463}=3.67, P<0.001$, for developmental time). Separating results by set enabled replicate estimation of variance components and heritability $\left(h^{2}\right)$, and therefore computation of parametric confidence limits around each estimate, rather than reliance on a theoretical variance determined from the sample size.
Table 1 Estimation of additive genetic variation $\left(S_{\mathrm{A}}^{2}\right)$ for resistance to heat shock in Drosophila buzzatii from ANOva (MS line by treatment $-\mathrm{MSE}) / 4$, from which repeatability (the intraclass correlation, $t)$ was calculated as $S_{\mathrm{A}}^{2} /\left(S_{\mathrm{A}}^{2}+\mathrm{MSE}\right)$. The isofemale heritability $(H)$ and narrow-sense heritability $\left(h^{2}\right)$ were estimated from this intraclass correlation, correcting for measurements on groups (Hoffmann \& Parsons, 1988). Because not all larvae develop to adults, we used the survivorship at $25^{\circ} \mathrm{C}$ for each set as the group correction factor for survival data and the proportion emerging after heat shock as the correction for developmental time data

\begin{tabular}{|c|c|c|c|c|}
\hline & Set 1 & Set 2 & Set 3 & Set 4 \\
\hline \multicolumn{5}{|c|}{ Survival with heat treatment } \\
\hline$S_{\mathrm{A}}^{2}$ & $0.0053^{* *}$ & $0.0037 *$ & $0.0087 * *$ & $<0$ \\
\hline MSE & 0.0189 & 0.0220 & 0.0337 & 0.0450 \\
\hline$t$ & 0.217 & 0.145 & 0.206 & - \\
\hline$H$ & 0.015 & 0.006 & 0.009 & - \\
\hline$h^{2}$ & 0.030 & 0.012 & 0.018 & - \\
\hline \multicolumn{5}{|c|}{ Developmental time after heat treatment } \\
\hline$S_{\mathrm{A}}^{2}$ & 0.0695 & $<0$ & 0.0132 & $0.1276^{* * *}$ \\
\hline MSE & 0.3654 & 0.2060 & 0.2755 & 0.2385 \\
\hline$t$ & 0.160 & - & 0.046 & 0.349 \\
\hline$H$ & 0.030 & - & 0.004 & 0.034 \\
\hline$h^{2}$ & 0.059 & - & 0.008 & 0.068 \\
\hline
\end{tabular}

The mean $h^{2}$ for survival was 0.015 with a $95 \%$ confidence interval lower limit of 0.003 and an upper limit of 0.027 ; for developmental time, the mean $h^{2}$ was 0.034 and the $95 \%$ confidence interval had lower and upper limits of 0.001 and 0.068 , respectively. However, two potential biases exist that may cause overestimation of these heritability estimates. The first is that these calculations of $S_{\mathrm{A}}^{2}$ from isofemale lines did not partition dominance and epistatic components of variation. Where dominance and epistasis are not explicitly estimated, part of the variation caused by these effects, if present, may add to the estimate of $S_{\mathrm{A}}^{2}$ and part may add to the environmental variance. The second potential bias is that components of variance used to estimate heritability for both survival and developmental time are constrained to zero as a lower limit. Consequently, averages among groups (Table 1) were calculated only from positive and zero values.

Variation within lines increased significantly following the stress treatment (Table 2); for survival in all four sets, and for developmental time in all but set 4 , the MSE increased significantly after stress (by $F$-tests of variance, $P<0.05)$. By contrast, among-line variation remained similar between treatments (variation twice was higher among lines after stress than in its absence, but in the other two groups, higher variation among 
Table 2 Summary ANOvas for line effects for the proportion of adult Drosophila buzzatii emerging (Survival) and mean developmental time (DT) for larvae reared in standard laboratory conditions $\left(25^{\circ} \mathrm{C}\right)$ and for those exposed to a $39^{\circ} \mathrm{C}$, $6 \mathrm{~h}$ heat shock, but otherwise reared at $25^{\circ} \mathrm{C}$. F-tests compare within-line variances in the presence and absence of heat shock

\begin{tabular}{|c|c|c|c|c|c|}
\hline & d.f. & Survival $25^{\circ} \mathrm{C}$ & DT $25^{\circ} \mathrm{C}$ & Survival heat shock & DT heat shock \\
\hline \multicolumn{6}{|l|}{ Set 1} \\
\hline Line effect & 23 & $0.0398 * * *$ & $0.551 * *$ & 0.0284 & 0.695 \\
\hline \multicolumn{6}{|l|}{ Set 2} \\
\hline Line effect & 22 & 0.0128 & $0.544 * * *$ & 0.0529 & $0.534^{*}$ \\
\hline \multicolumn{6}{|l|}{ Set 3} \\
\hline Line effect & 24 & $0.0816 * * *$ & $0.585 * * *$ & 0.0588 & $0.678 *$ \\
\hline MSE & 71 & 0.0195 & 0.191 & $\begin{array}{l}0.0479 \\
\left(F=2.5^{* *}\right)\end{array}$ & $\begin{array}{l}0.379 \\
\left(F=2.0^{*}\right)\end{array}$ \\
\hline \multicolumn{6}{|l|}{ Set 4} \\
\hline
\end{tabular}

$* P<0.05, * * P<0.01, * * * P<0.001$

lines occurred in the nonstress treatment). The consequence of the higher within-line variation after stress was that significance of the among-line effect was limited to the nonstress group (Table 2), but, because amongline variation was not lost, tests of correlation coefficients among variables with and without stress compare trait means that vary similarly among lines.

Lines of $D$. buzzatii also varied significantly in fecundity and longevity at a constant $25^{\circ} \mathrm{C}$. Because these traits were measured after more than five generations in the laboratory, variance components cannot be estimated accurately. However, little research exists on longevity in Drosophila species other than D. melanogaster, and therefore Appendix 1 provides a life table for D. buzzatii at $25^{\circ} \mathrm{C}$ that includes standard errors among lines for each estimated value. All standard measurements of longevity were highly correlated, and therefore we presented mean life span in the correlation analyses among traits.

\section{Correlation coefficients among traits}

Correlation analyses among the seven traits produced 21 coefficients, or twice this number given separate analyses for females and males. However, a single experimentwide test on these data is unduly conservative, because genetic correlations between life-history traits are predicted not to be high. Instead, correlation coefficients are presented whenever results were consistent in males and females, and where either one coefficient was significant at $P<0.05$ and/or where the combined probability across genders was significant (Fig. 1). Seven comparisons satisfied these criteria.

Four of the seven correlations that were largest in magnitude involved larval developmental time at $25^{\circ} \mathrm{C}$. Three coefficients that involved developmental time were positive (as a fitness relationship), those with survival and with developmental time after larval heat shock and with adult longevity, but shorter developmental time associated with smaller fecundity rates (short developmental time is considered a fitness advantage relative to slow development, and therefore the sign of the relationship for all coefficients involving developmental time in Fig. 1 were reversed to reflect fitness correlations rather than the purely numerical relationships using the raw results). Higher survivorship at $25^{\circ} \mathrm{C}$ also associated with reduced fecundity, and adult longevity and survival after larval heat-shock correlated significantly and positively. However, these last two relationships occurred predominantly in data of only males or only females, and were weak in the other gender. Coefficients with adult thermotolerance were not significant in any test for correlation among traits.

\section{Discussion}

The response of larvae to heat is heritable, but the largest estimate of heritability for survival following heat shock was 0.030 , whereas that for the delay induced in developmental time showed a maximum of 0.068 within the four independent sets of lines. Lower limits for these heritabilities approached zero for both traits, 


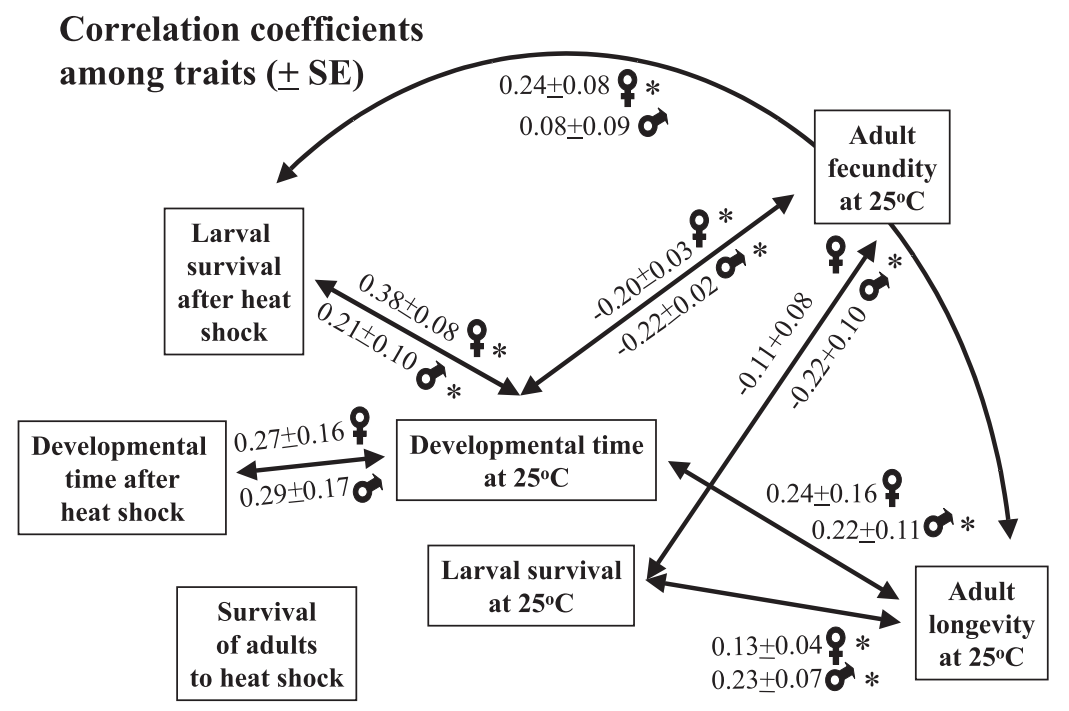

Fig. 1 The genetic relationships among seven traits in Drosophila buzzatii related to fitness: larval survival and developmental time at $25^{\circ} \mathrm{C}$, the same traits in larvae also exposed to a $39^{\circ} \mathrm{C}$ heat shock, survival of adults to heat shock (data from Krebs \& Loeschcke, 1997), adult fecundity at $25^{\circ} \mathrm{C}$ and longevity at $25^{\circ} \mathrm{C}$. Lines connect those traits that correlated significantly in one or both sexes, or where the combined probability for males and females was significant (individual significance occurs where $r$ equals or exceeds $1.96 \times \mathrm{SE}$, and is indicated by *). Each coefficient and its standard error were determined from the means of four independent sets of 25 lines. Because short developmental time is considered a fitness advantage as compared to slow development, all relationships reflect fitness correlations with developmental time rather than a strict numerical representation (which would be the coefficient times -1$)$.

which indicates a wide range of low possible values, similar to that found for adult thermotolerance (Krebs \& Loeschcke, 1997). However, these results in larvae may be conservative because we assumed complete density dependence when accounting for variation caused by different numbers of flies emerging from vials, because a density-dependent model of within-line variance was empirically demonstrated in assays of adult thermotolerance (Krebs \& Loeschcke, 1997). With complete density-dependent effects, the within-line variance is multiplied by the group size to estimate the intraclass correlation (Hoffmann \& Parsons, 1988), but, if incorrect, the intraclass correlation and all values derived from it will be underestimated.

In contrast to our thermotolerance measures, fecundity, longevity, and developmental time (as traits themselves, rather than their changes in response to heat) typically show much higher estimates of heritability under controlled laboratory conditions (Rose \& Charlesworth, 1981). Because data on these traits came after more than five laboratory generations, and inbreeding or drift in this time may bias between-line variance components (Hoffmann \& Parsons, 1988), heritabilities were not estimated for them. However, the correlation coefficients that included fecundity, longevity and particularly developmental time, where several significant effects occurred (Fig. 1), remain qualitatively robust.

Developmental time at $25^{\circ} \mathrm{C}$ predominated within a complex set of interactions with the other life-history traits. Most relationships were positive, but fecundity correlated negatively with developmental time and with survival. In contrast, genetic relationships between developmental time of $D$. buzzatii larvae that survived the stress appeared much smaller, and only the crossenvironment correlation with developmental time at $25^{\circ} \mathrm{C}$ remained significant. Larval survival showed no cross-environment relationship, and adult thermotolerance did not covary with any of the traits measured here, nor with adult metabolic rate (Loeschcke et al., 1997). However, the heat stress revealed one new association between traits. Larvae in lines that developed faster at $25^{\circ} \mathrm{C}$ survived the stress in higher proportions, a positive relationship across these two environments.

By using large sample sizes, tests for correlations were powerful, and as shown previously by Krebs \& Feder (1997a), these isofemale-line techniques adequately assess relationships with threshold traits like survival. Therefore, lack of correlations and/or their disappearance after treatment are attributable to the stress. In fact, previous comparisons among populations of D. buzzatii support the results found here. As in the 
isofemale lines, larval and adult stress tolerance varied greatly among populations, but thermotolerance between stages was unrelated (Krebs \& Loeschcke, 1995). Coyne et al. (1983) similarly found that for seven D. pseudoobscura populations, relative tolerance differed between the adult and pupal stages. Population studies on D. buzzatii also agree for the association between shorter larva-to-adult developmental time at $25^{\circ} \mathrm{C}$ and higher larval thermotolerance as measured by survival (Krebs \& Loeschcke, 1995), a result independently obtained after selection on thermotolerance (Loeschcke \& Krebs, 1996). Perhaps shorter developmental time contributes to higher larval resistance after heat stress because a higher metabolic rate facilitates more rapid acclimation to the rise in temperature. Hard evidence of a mechanism, unfortunately, does not exist.

Although the genes underlying most life-history traits are unknown, physiological stress can quantitatively change genetic correlations among these traits (Hoffmann \& Parsons, 1991). One potential mechanism for this change is that stress reveals hidden variation by breaking down homeostatic processes (Neyfakh \& Hartl, 1993). Rutherford \& Lindquist (1998) propose that stress may also overwhelm developmental buffers, because many mutant alleles, which have no effect on the phenotype under normal conditions because of canalization, may contribute to variation in development after molecular chaperones like Hsp90 are shunted to other duties within the cell. That heat stress increased environmental (within-line) variance more than additive genetic (between-line) variance, is compatible with a model where stress imposes stochastic affects on homeostasis among individuals of all families. This result also indicates why the influence of environmental heterogeneity, even when the environmental change is a single heat shock, is difficult to predict: heritabilities may either increase or decrease, depending on the relative change in the genetic and environmental components of variance.

Although these experiments demonstrate several significant trait correlations, one negative coefficient expected was not strongly observed in D. buzzatii, that between fecundity and longevity. This trade-off is one of the best established negative life-history relationships in D. melanogaster (Partridge \& Fowler, 1992; references therein). Although inbreeding, or line variation for a nonspecific advantage in the novel laboratory environment, can mask trade-offs (Service \& Rose, 1985), the rapid analysis of each trait soon after originating the lines and the low-density rearing largely reduced this problem, as predicted (Latter \& Mulley, 1995). Furthermore, larval developmental time and survival at $25^{\circ} \mathrm{C}$ both correlated negatively with fecundity, which provides strong support that these potential confound- ing effects were minimized. Thus, we may conclude that, in D. buzzatii, fecundity and longevity associate only indirectly through developmental time. Likewise, larval thermotolerance and survival in the absence of stress showed no relationship, although the two traits were found to be negatively correlated in D. melanogaster when isofemale line methods were also used (Krebs \& Feder, 1997a).

Our results demonstrate that stress alters covariance patterns among traits, as may other forms of habitat variation (Kirkpatrick, 1996). The task for the future is to clarify how these puzzling shifts in genetic relationships occur. One approach is to identify the underlying physiological responses to change. For thermal stress, analysis of Hsp70 is paying dividends. Variation in Hsp70 expression affects thermotolerance in D. melanogaster (Feder et al., 1996; Dahlgaard et al., 1998) and D. buzzatii (Loeschcke \& collaborators, unpublished data). Variation in Hsp70 expression also may affect survival and developmental time (Krebs \& Feder, 1997b) and, surprisingly, longevity in D. melanogaster (Tatar et al., 1997). Therefore, that stress may alter trade-offs and other trait associations, and the potential for Hsp70 variation to underlie these changes, opens the door for investigation of the actual genes that may control variation in life-history traits, particularly in populations that experience environmental extremes.

\section{Acknowledgements}

We thank Camilla Håkansson, Birgit Sørensen and Doth Andersen for their valuable assistance with data collection. This research was made possible by grants to V.L. from the Carlsberg Foundation (no. 93-0280-30) and the Danish Natural Science Research Council, which also supported R.K.'s stay in Denmark.

\section{References}

BARKeR, J. S. F. 1992. Genetic variation in cactophilic Drosophila for oviposition on natural yeast substrates. Evolution, 46, 1070-1083.

BIJLSMA, R. AND LOESCHCKE, v. (eds) 1997. Environmental Stress, Adaptation and Evolution. Birkhäuser-Verlag, Basel.

COLEMAN, J. S., HECKATHORN, S. A. AND HALlBERG, R. L. 1995. Heat-shock proteins and thermotolerance: linking ecological and molecular perspectives. Trends Ecol. Evol., 10, 305-306. COYNE, J. A., BUNDGAARD, J. AND PROUT, T. 1983. Geographic variation of tolerance to environmental stress in Drosophila pseudoobscura. Am. Nat., 122, 474-488.

DAHLGAARD, J., LOESCHCKE, V., MiCHALAK, P. AND JUSTESEN, J. 1998. Acclimation, thermal resistance and associated expression of the heat-shock protein, Hsp70, in adult Drosophila melanogaster. Funct. Ecol., 12, 786-793. 
DAVID, J. R., ALLEMAND, R., VAN HERREWEGE, J. AND COHET, Y. 1983. Ecophysiology: abiotic factors. In: Ashburner, M. Carson, H. L. and Thompson, J. R., Jr. (eds) The Genetics and Biology of Drosophila, vol. 3d, pp. 105-170. Academic Press, London.

FEDER, M. E. 1996. Ecological and evolutionary physiology of stress proteins and the stress response: the Drosophila melanogaster model. In: Johnston, I. A. and Bennett, A. F. (eds) Phenotypic and Evolutionary Adaptations to Temperature, pp. 79-102. SEB Seminar Series, Cambridge University Press, Cambridge.

FEDER, M. E., CARTAÑO, N. V., MILOS, L., KREBS, R. A. AND LINDQUiST, S. L. 1996. Effect of engineering Hsp70 copy number on Hsp70 expression and tolerance of ecologically relevant heat shock in larvae and pupae of Drosophila melanogaster. J. Exp. Biol., 199, 1837-1844.

HEDRICK, P. W. 1986. Genetic polymorphism in heterogeneous environments. Ann. Rev. Ecol. Syst., 7, 1-32.

HOFFMANN, A. A. 1995. Acclimation: increasing survival at a cost. Trends Ecol. Evol., 10, 1-2.

HOFFMANN, A. A. AND PARSONS, P. A. 1988. The analysis of quantitative variation in natural populations with isofemale strains. Génét. Sél. Évol., 20, 87-98.

HOFFMANN, A. A. AND PARSONS, P. A. 1991. Evolutionary Genetics and Environmental Stress. Oxford Science Publications, Oxford.

HOFFMANN, A. A. AND PARSONS, P. A. 1997. Extreme Environmental Change and Evolution. Cambridge University Press, Cambridge.

HUEY, R. B. AND BENNETT, A. F. 1990. Physiological adjustments to fluctuating thermal environments: an ecological and evolutionary perspective. In: Morimoto, R. I., Tissiéres, A. and Georgopoulos, C. (eds) Stress Proteins in Biology and Medicine, pp. 37-59. Cold Spring Harbor Laboratory Press, Cold Spring Harbor, NY.

JOHnStON, I. A. AND BENNETT, A. F. (eds) 1996. Phenotypic and Evolutionary Adaptations to Temperature. SEB Seminar Series, Cambridge University Press, Cambridge.

KIRKPATRICK, M. 1996. Genes and adaptation: a pocket guide to the theory. In: Rose, M. R. and Lauder, G. V. (eds) Adaptation, pp. 125-146. Academic Press, San Diego, CA.

KREBS, R. A. AND FEDER, M. E. 1997a. Natural variation in the expression of the heat-shock protein $\mathrm{Hsp} 70$ in a population of Drosophila melanogaster, and its correlation with tolerance of ecologically relevant thermal stress. Evolution, 51, 173-179.

KREBS, R. A. AND FEDER, M. E. 1997b. Deleterious consequences of Hsp70 overexpression in Drosophila melanogaster larvae. Cell Stress \& Chaperones, 2, 60-71.

KREBS, R. A. AND LOESCHCKE, V. 1994. Costs and benefits of activation of the heat-shock response in Drosophila melanogaster. Funct. Ecol., 8, 730-737.
KREBS, R. A. AND LOESCHCKE, V. 1995. Resistance to thermal stress in preadult Drosophila buzzatii: variation among populations and changes in relative resistance across life stages. Biol. J. Linn. Soc., 56, 517-531.

KREBS, R. A. AND LOESCHCKE, v. 1997. Estimating heritability in a threshold trait: variation in heat stress resistance among isofemale lines of Drosophila buzzatii. Heredity, 79, 252259.

LATTER, B. D. H. AND MULLEY, J. C. 1995. Genetic adaptation to captivity and inbreeding depression in small laboratory populations of Drosophila melanogaster. Genetics, 139, 255266.

LOESCHCKE, V. AND KREBS, R. A. 1996. Selection for heat-shock resistance in larval and adult Drosophila buzzatii: comparing direct and indirect responses on viability and development. Evolution, 50, 2354-2359.

LOESCHCKE, V., KREBS, R. A., DAHGAARD, J. AND MICHALAK, P. 1997. High-temperature stress and the evolution of thermal resistance in Drosophila. In: Bijlsma, R. and Loeschcke, V. (eds) Environmental Stress, Adaptation and Evolution, pp. 175-190. Birkhäuser-Verlag, Basel.

NEYFAKH, A. A. AND HARTL, D. L. 1993. Genetic control of the rate of embryonic development: selection for faster development at elevated temperatures. Evolution, 47, 16251631.

PARSEll, D. A. AND LINDQUist, s. 1993. The function of heatshock proteins in stress tolerance: degradation and reactivation of damaged proteins. Ann. Rev. Genet., 27, 437496.

PARTRIDGE, L. AND FOWLER, K. 1992. Direct and correlated responses to selection at age of reproduction in Drosophila melanogaster. Evolution, 46, 76-91.

ROSE, M. R. AND CHARLESWORTH, B. 1981. Genetics of lifehistory in Drosophila melanogaster. I. Sib analysis of adult females. Genetics, 97, 187-196.

RUTHERFORD, S. L. AND LINDQUiST, S. 1998. Hsp90 as a capacitor for morphological evolution. Nature, 396, 336342.

SERVICE, P. M. AND ROSE, M. R. 1985. Genetic covariation among life-history components: the effect of novel environments. Evolution, 39, 943-945.

SGRO, C. M. AND HOFFMANN, A. A. 1998. Heritable variation for fecundity in field-collected Drosophila melanogaster and their offspring reared under different environmental temperatures. Evolution, 52, 134-143.

SOLOMON, J. M., ROSSI, J. M., GOLIC, K., MCGARRY, T. AND LINDQUIST, S. 1991. Changes in Hsp70 alter thermotolerance and heat-shock regulation in Drosophila. The New Biologist, 3, 1106-1120.

TATAR, M., KHAZAeli, A. A. AND CURTSinger, J. W. 1997. Chaperoning extended life. Nature, 390, 30. 


\section{Appendix 1}

Life table for (a) females and (b) males of Drosophila buzzatii held with members of the opposite sex at a constant $25^{\circ} \mathrm{C}$. Means among lines are presented $\pm 1 \mathrm{SE}$ for $l_{x}$ (the percentage surviving to each age group), ${ }_{n} d_{x}$ (the percentage in that age group that died), ${ }_{n} q_{x}$ (the cumulative percentage that died), ${ }_{n} L_{x}=7 \times\left[1 / 2\left(l_{x}+l_{x+1}\right)\right]$ (the number alive during each age class multiplied by the days within each class), $T_{x}=\left(T_{x+1}+L_{x}\right)$ (an intermediate step in the calculation of life expectancy) and the expected days to live, $e=T_{x / n} L_{x}$. $N$ is the number of lines where one or more individuals remained alive, and therefore the estimates of expected lifespan apply only to those lines

\begin{tabular}{|c|c|c|c|c|c|c|c|}
\hline Age & $l_{x}$ & ${ }_{n} d_{x}$ & ${ }_{n} q_{x}$ & ${ }_{n} L_{x}$ & $T_{x}$ & $e$ & $N$ \\
\hline \multicolumn{8}{|c|}{ (a) Females } \\
\hline 0 & 100 & $1.6 \pm 0.2$ & $1.6 \pm 0.2$ & $694.4 \pm 0.5$ & $4098.7 \pm 67.2$ & $41.0 \pm 0.7$ & 98 \\
\hline 7 & $98.4 \pm 0.2$ & $1.5 \pm 0.2$ & $3.1 \pm 0.2$ & $683.6 \pm 1.3$ & $3404.4 \pm 67.1$ & $34.5 \pm 0.7$ & 98 \\
\hline 14 & $97.0 \pm 0.2$ & $4.1 \pm 0.5$ & $7.2 \pm 0.6$ & $664.1 \pm 2.6$ & $2720.8 \pm 66.5$ & $27.9 \pm 0.7$ & 98 \\
\hline 21 & $92.8 \pm 0.6$ & $9.9 \pm 1.2$ & $17.1 \pm 1.6$ & $615.2 \pm 7.5$ & $2056.7 \pm 64.6$ & $21.7 \pm 0.6$ & 98 \\
\hline 28 & $82.9 \pm 1.6$ & $10.8 \pm 0.8$ & $27.8 \pm 2.2$ & $542.9 \pm 13.3$ & $1441.6 \pm 58.1$ & $16.3 \pm 0.5$ & 98 \\
\hline 3 & $72.2 \pm 2.2$ & $19.3 \pm 1.0$ & $47.1 \pm 2.5$ & $437.7 \pm 15.9$ & $898.6 \pm 46.4$ & $11.3 \pm 0.4$ & 98 \\
\hline 42 & $52.9 \pm 2.5$ & $24.2 \pm 1.2$ & $71.3 \pm 2.3$ & $285.5 \pm 16.1$ & $460.9 \pm 32.0$ & $7.4 \pm 0.3$ & 98 \\
\hline 49 & $28.7 \pm 2.3$ & $19.2 \pm 1.6$ & $90.5 \pm 1.2$ & $133.8 \pm 11.8$ & $175.4 \pm 17.2$ & $5.1 \pm 0.2$ & 97 \\
\hline 56 & $9.5 \pm 1.2$ & $8.5 \pm 1.1$ & $99.0 \pm 3.0$ & $36.9 \pm 5.1$ & $41.6 \pm 6.2$ & $4.1 \pm 0.2$ & 75 \\
\hline 63 & $1.0 \pm 0.3$ & $0.9 \pm 0.2$ & $99.9 \pm 0.1$ & $4.0 \pm 1.2$ & $4.8 \pm 1.6$ & $3.8 \pm 0.2$ & 28 \\
\hline 70 & $0.1 \pm 0.1$ & $0.1 \pm 0.04$ & $100.0 \pm 0.00$ & $0.6 \pm 0.4$ & $0.7 \pm 0.5$ & $4.5 \pm 1.0$ & 4 \\
\hline 77 & $0.04 \pm 0.04$ & 0.04 & 100.0 & $0.1 \pm 0.1$ & $0.1 \pm 0.1$ & 3.5 & 1 \\
\hline \multicolumn{8}{|c|}{ (b) Males } \\
\hline 0 & 100 & $2.2 \pm 0.2$ & $2.2 \pm 0.2$ & $692.2 \pm 0.7$ & $4268.3 \pm 71.0$ & $42.7 \pm 0.7$ & 98 \\
\hline 7 & $97.8 \pm 0.2$ & $1.5 \pm 0.2$ & $3.8 \pm 0.3$ & $679.1 \pm 1.5$ & $3576.1 \pm 70.9$ & $36.6 \pm 0.7$ & 98 \\
\hline 14 & $96.2 \pm 0.3$ & $4.8 \pm 0.5$ & $8.5 \pm 0.6$ & $657.0 \pm 2.7$ & $2897.0 \pm 70.6$ & $30.1 \pm 0.7$ & 98 \\
\hline 21 & $91.5 \pm 0.6$ & $9.8 \pm 1.2$ & $18.4 \pm 1.6$ & $605.7 \pm 7.2$ & $2240.1 \pm 68.9$ & $24.1 \pm 0.7$ & 98 \\
\hline 28 & $81.6 \pm 1.6$ & $8.8 \pm 0.7$ & $27.2 \pm 2.1$ & $540.4 \pm 12.6$ & $1634.3 \pm 62.7$ & $19.2 \pm 0.5$ & 98 \\
\hline 35 & $72.8 \pm 2.1$ & $15.4 \pm 0.9$ & $42.6 \pm 2.3$ & $455.8 \pm 14.9$ & $1093.9 \pm 51.9$ & $14.2 \pm 0.4$ & 98 \\
\hline 42 & $57.4 \pm 2.3$ & $21.1 \pm 1.0$ & $63.7 \pm 2.3$ & $328.0 \pm 15.5$ & $638.2 \pm 38.7$ & $10.2 \pm 0.3$ & 98 \\
\hline 49 & $36.3 \pm 2.3$ & $18.8 \pm 1.2$ & $82.5 \pm 1.6$ & $188.3 \pm 13.0$ & $310.2 \pm 24.5$ & $8.2 \pm 0.3$ & 98 \\
\hline 56 & $17.5 \pm 1.6$ & $11.5 \pm 1.0$ & $94.0 \pm 0.7$ & $82.2 \pm 7.8$ & $121.9 \pm 12.3$ & $6.9 \pm 0.3$ & 95 \\
\hline 63 & $6.0 \pm 0.7$ & $4.0 \pm 0.5$ & $98.0 \pm 0.3$ & $27.9 \pm 3.3$ & $39.7 \pm 4.9$ & $6.7 \pm 0.3$ & 80 \\
\hline 70 & $2.0 \pm 0.3$ & $1.4 \pm 0.2$ & $99.5 \pm 0.1$ & $8.8 \pm 1.2$ & $11.8 \pm 1.7$ & $6.1 \pm 0.5$ & 63 \\
\hline 77 & $0.5 \pm 0.1$ & $0.4 \pm 0.1$ & $99.8 \pm 0.0$ & $2.5 \pm 0.5$ & $3.1 \pm 0.6$ & $5.5 \pm 0.5$ & 30 \\
\hline 84 & $0.2 \pm 0.05$ & 0.2 & 100.0 & $0.6 \pm 0.2$ & $0.6 \pm 0.2$ & 3.5 & 12 \\
\hline
\end{tabular}

(c) The Genetical Society of Great Britain, Heredity, 83, 46-53. 